\title{
Fermi Surface-Brillouin Zone Interactions in 2/1-2/1-2/1 Bergman-Type Approximant $\mathrm{Na}_{27} \mathrm{Au}_{27} \mathrm{Ga}_{31}$
}

 \\ AND G.J. MilleR ${ }^{e}$ \\ ${ }^{a}$ Nagoya Industrial Science Research Institute, 1-13 Yotsuya-dori, Chikusa-ku, Nagoya, 464-0819, Japan \\ ${ }^{b}$ Aichi University of Education, Kariya-shi, Aichi, 448-8542, Japan \\ ${ }^{c}$ Nagoya Institute of Technology, Department of Frontier Materials, Gokiso-cho, Showa-ku, Nagoya 466-8555, Japan \\ ${ }^{d}$ Theoretical Physics, University of Kassel, 34132 Kassel, Germany \\ ${ }^{e}$ Ames Laboratory, US-DOE \& Department of Chemistry, Iowa State University, U.S.A.
}

\begin{abstract}
The X-ray diffraction studies on a newly synthesized $\mathrm{Na}_{26} \mathrm{Au}_{25} \mathrm{Ga}_{29}$ single crystal revealed the formation of a single phase 2/1-2/1-2/1 Bergman-type approximant and the presence of Au/Ga mixed occupancies in its unit cell containing 680 atoms. The structure model of the 2/1-2/1-2/1 approximant with composition $\mathrm{Na}_{27} \mathrm{Au}_{27} \mathrm{Ga}_{31}$ was constructed by eliminating the chemical disorder with a minimum sacrifice of composition displacement. The full potential linearized augmented plane wave electronic structure calculations with subsequent full potential linearized augmented plane wave-Fourier analysis were performed for the 2/1-2/1-2/1 approximant $\mathrm{Na}_{27} \mathrm{Au}_{27} \mathrm{Ga}_{31}$ with space group $\mathrm{Pa} \overline{3}$. The square of the Fermi diameter $\left(2 k_{\mathrm{F}}\right)^{2}$, electrons per atom ratio $e / a$ and the critical reciprocal lattice vector $|\boldsymbol{G}|^{2}$ are determined. A shallow pseudogap at the Fermi level was interpreted as originating from interference of electrons having $\left(2 k_{\mathrm{F}}\right)^{2}=109.2 \pm 1.0$ with sets of lattice planes with $|\boldsymbol{G}|^{2}$ 's centered at 108 . The effective $e / a$ value for the compound is found to be 1.76 in good agreement with 1.73 derived from a composition average of $(e / a)_{\mathrm{Na}}=1.0,(e / a)_{\mathrm{Au}}=1.0$ and $(e / a)_{\mathrm{Ga}}=3.0$.
\end{abstract}

DOI: $10.12693 /$ APhysPolA.126.535

PACS: 71.23.Ft, 71.20.Dg, 71.15.Mb

\section{Introduction}

Through systematic exploration of the $\mathrm{Na}-\mathrm{Au}-\mathrm{Ga}$ system, Lin and his coworkers have discovered a series of structurally complex metallic alloy (CMA) phases including an icosahedral quasicrystal $\mathrm{Na}_{13} \mathrm{Au}_{12} \mathrm{Ga}_{15}$, an orthorhombic approximant $\mathrm{Na}_{32} \mathrm{Au}_{38} \mathrm{Ga}_{30}$, two structurally related Bergman-type 1/1-1/1-1/1 approximants $\mathrm{Na}_{26} \mathrm{Au}_{36} \mathrm{Ga}_{19}$ and $\mathrm{Na}_{26} \mathrm{Au}_{18} \mathrm{Ga}_{36}$ and the Bergmantype 2/1-2/1-2/1 approximant $\mathrm{Na}_{26} \mathrm{Au}_{25} \mathrm{Ga}_{29}$ [1, 2]. By studying the total crystal orbital Hamilton population (COHPs) in the framework of linear muffin-tin (MT) orbital in the atomic sphere approximation (LMTO-ASA) band calculations, they pointed out the presence of strong covalent bonding in both $\mathrm{Ga}-\mathrm{Au}$ and $\mathrm{Na}-\mathrm{Au}$ interactions in the model 1/1-1/1-1/1 approximant $\mathrm{Na}_{26} \mathrm{Au}_{24} \mathrm{Ga}_{30}$ and took it as a characteristic feature of the polar intermetallics in spite of the possession of low electrons per atom ratio $e / a$ equal to 1.75 . It is, therefore, of great interest to investigate whether the Hume-Rothery stabilization mechanism is effective in such polar CMAs.

Mizutani and coworkers [3-8] employed first-principles full potential linearized augmented plane wave (FLAPW) electronic structure calculations and established a powerful technique to extract Fermi surface-Brillouin zone $(\mathrm{FsBz})$ interactions associated with the interference phe-

*corresponding author; e-mail: uichiro@xa3.so-net.ne.jp nomenon and to determine the $e / a$ value for elements and intermetallic compounds, regardless of whether a transition metal (TM) element is involved or not. They have made full use of its formalism, in which the wave functions outside the MT spheres are expanded into plane waves over allowed reciprocal lattice vectors $\boldsymbol{G}$.

Prior to the discovery of the Bergman-type 2/1-2/12/1 approximant $\mathrm{Na}_{26} \mathrm{Au}_{25} \mathrm{Ga}_{29}$, Lin and Corbett [9] had already identified the $\mathrm{Mg}_{27} \mathrm{Al}_{10.7} \mathrm{Zn}_{47.3}$ compound to be a Bergman-type 2/1-2/1-2/1 approximant with space group $\mathrm{Pa} \overline{3}$. Encouraged by their pioneering work, we could refine the crystallographic data for the 2/1-2/1-2/1 approximant $\mathrm{Na}_{26} \mathrm{Au}_{25} \mathrm{Ga}_{29}$ from both powder and single crystal X-ray diffraction studies. After constructing its model structure free from chemical disorder, the FLAPW band calculations with the subsequent FLAPW-Fourier analysis was performed. To the best of our knowledge, first-principles FLAPW band calculations have been carried out for the first time for a 2/1-2/1-2/1 approximant.

\section{Atomic structure determinations and electronic structure calculations}

It was revealed that the $\mathrm{Na}_{26} \mathrm{Au}_{25} \mathrm{Ga}_{29}$ compound crystallizes into a Bergman-type 2/1-2/1-2/1 approximant containing 680 atoms per unit cell with space group $P a \overline{3}$ and lattice constant of $23.446 \AA$. A structure refinement led us to conclude that the compound is free from chemical disorder except for $\mathrm{Au} / \mathrm{Ga}$ mixed site occupancies. To facilitate FLAPW band calculations, we constructed the model structure $\mathrm{Na}_{27} \mathrm{Au}_{27} \mathrm{Ga}_{31}$ by eliminating the $\mathrm{Au} / \mathrm{Ga}$ chemical disorder from the refined 
crystallographic data, which will be reported elsewhere.

FLAPW band calculations have been performed by employing the commercially available WIEN2k program package [10] with INTEL version Linux personal computers. The WIEN $2 \mathrm{k}$ was run by using the cut-off parameter $R_{\mathrm{MT}} K_{\max }=5.8$ and 6.2 , which determines the number of basis functions or size of the matrices, and the number of meshes, $N_{k}=216$ for $\mathrm{Na}_{27} \mathrm{Au}_{27} \mathrm{Ga}_{31}$. Our in-house Fortran90 Program was employed to carry out the FLAPWFourier analysis by using the "case.output1" file generated by running WIEN2k [3-8]. Briefly, the FLAPWFourier spectrum represents the energy dependence of the square of the Fourier coefficient of the plane wave specified by the square of reciprocal lattice vector $|\boldsymbol{G}|^{2}$ in units of $(2 \pi / a)^{2}$ at selected symmetry points of the Brillouin zone. One can extract electronic states dominating at the Fermi level from the energy spectrum. The $|\boldsymbol{G}|^{2}$ thus extracted is called critical. As another tool, the energy dispersion relation is constructed for electrons outside the MT spheres. We specifically call it the HumeRothery plot, since it allows us to determine the square of the effective Fermi diameter $\left(2 k_{\mathrm{F}}\right)^{2}$ and $e / a$ values for each intermetallic compound studied [3-8].

\section{Results and discussions}

The total DOS for $\mathrm{Na}_{27} \mathrm{Au}_{27} \mathrm{Ga}_{31}$ is shown in Fig. 1a and $\mathrm{b}$, where ordinates are shown on linear and logarithmic scales, respectively. As guided by a dotted line in Fig. 1b, a shallow pseudogap becomes noticeable and is found to be widely spread over -2 to $+3 \mathrm{eV}$ across the Fermi level $E_{\mathrm{F}}$.
A DOS pseudogap is hardly seen on linear scales. The DOS profile is very similar to that reported for the Bergman-type 1/1-1/1-1/1 model approximant $\mathrm{Na}_{26} \mathrm{Au}_{24} \mathrm{Ga}_{30}[1,2]$.

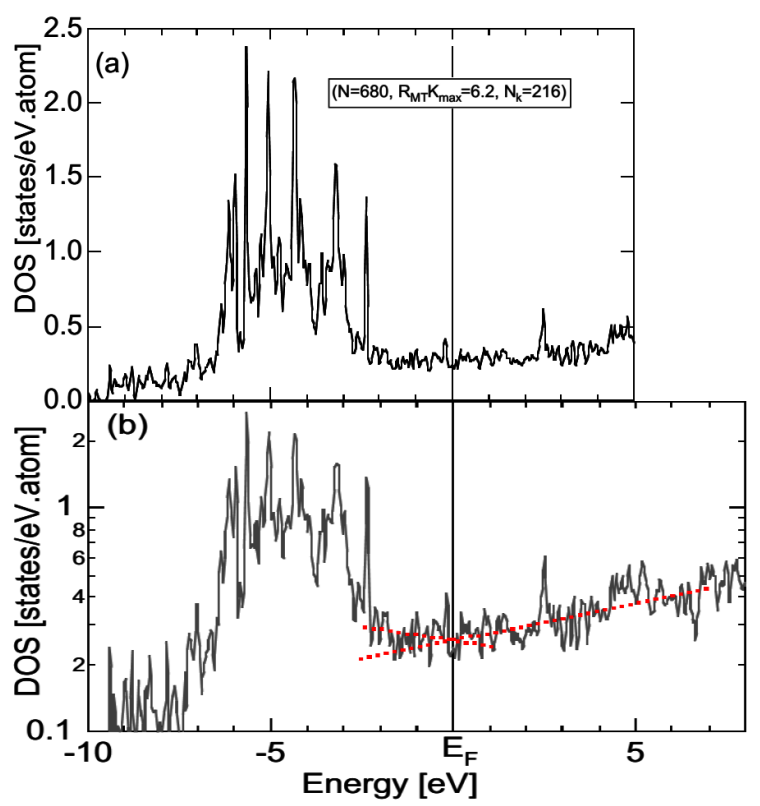

Fig. 1. The total DOS for the 2/1-2/1-2/1 approximant $\mathrm{Na}_{27} \mathrm{Au}_{27} \mathrm{Ga}_{31}$ on (a) the linear and (b) logarithmic scale for the ordinate. Dotted lines drawn in (b) are guide to the eye.

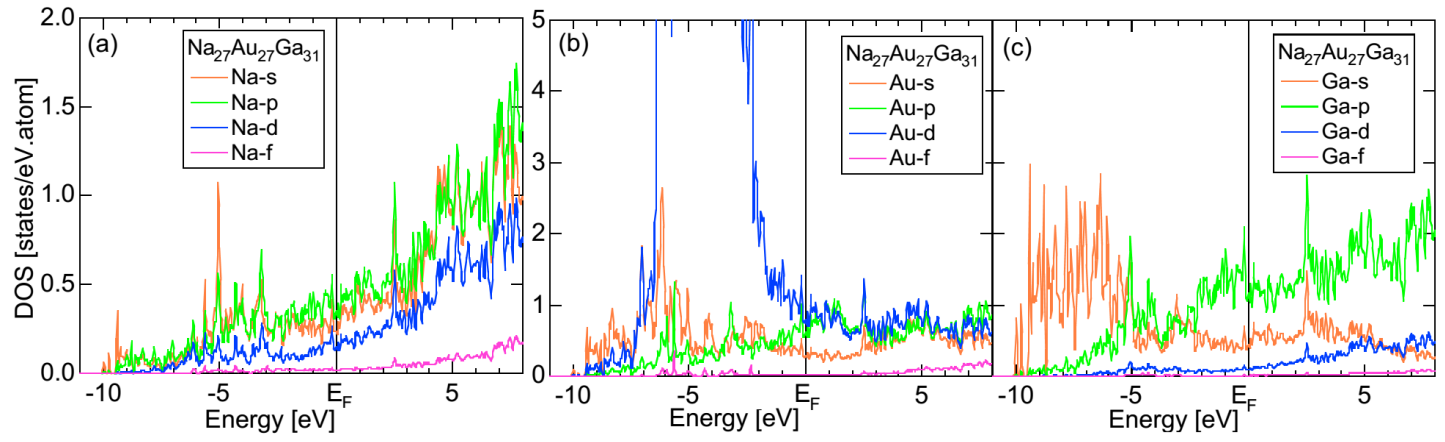

Fig. 2. (a) Na-, (b) Au- and (c) Ga-partial DOS of the 2/1-2/1-2/1 approximant $\mathrm{Na}_{27} \mathrm{Au}_{27} \mathrm{Ga}_{31}$.

The Na-, Au- and Ga-partial DOSs in $\mathrm{Na}_{27} \mathrm{Au}_{27} \mathrm{Ga}_{31}$ are shown in Fig. 2-c, respectively. A peak is found across $E_{\mathrm{F}}$ in the Ga-4p partial DOS. This is nothing but the formation of the anti-bonding Ga- $4 p$ states as a result of orbital hybridizations with $\mathrm{Au}-5 d$ states. This explains why the DOS pseudogap minimum at $E_{\mathrm{F}}$ is masked and lends support to the presence of strong covalent $\mathrm{Au}-\mathrm{Ga}$ bonding $[1,2]$. Now the origin of the shallow pseudogap is discussed in terms of $\mathrm{FsBz}$ interactions by constructing both FLAPW-Fourier spectrum and the Hume-Rothery plot.

Figure 3 shows the FLAPW-Fourier spectra at symmetry points $X, \Gamma$ and $R$ over the range $|\boldsymbol{G}|^{2}=90$ to 129 along with the total DOS for the $2 / 1-2 / 1-2 / 1$ approx- imant $\mathrm{Na}_{27} \mathrm{Au}_{27} \mathrm{Ga}_{31}$. One can immediately find that the center of gravity energy marked with green circles meets the Fermi level for LAPW waves of $|\boldsymbol{G}|^{2}=108$ at symmetry point $\Gamma$. More important is the fact that the Fourier coefficients remain finite at the Fermi level over a wide range of $|\boldsymbol{G}|^{2}$ from 96 to 126 . This is taken as the evidence for the participation of multi-zones in the interference phenomenon and also for the development of spatially localized polar interactions. Among them, $|\boldsymbol{G}|^{2}=108$ can be selected to be critical. It is emphasized that multi-zone effects are commonly observed in CMAs with a giant unit cell like the RT-type Al-Mg-Zn $1 / 1-1 / 1-1 / 1$ approximant and that only a shallow and widely spread pseudogap is formed $[3,4,6]$. 


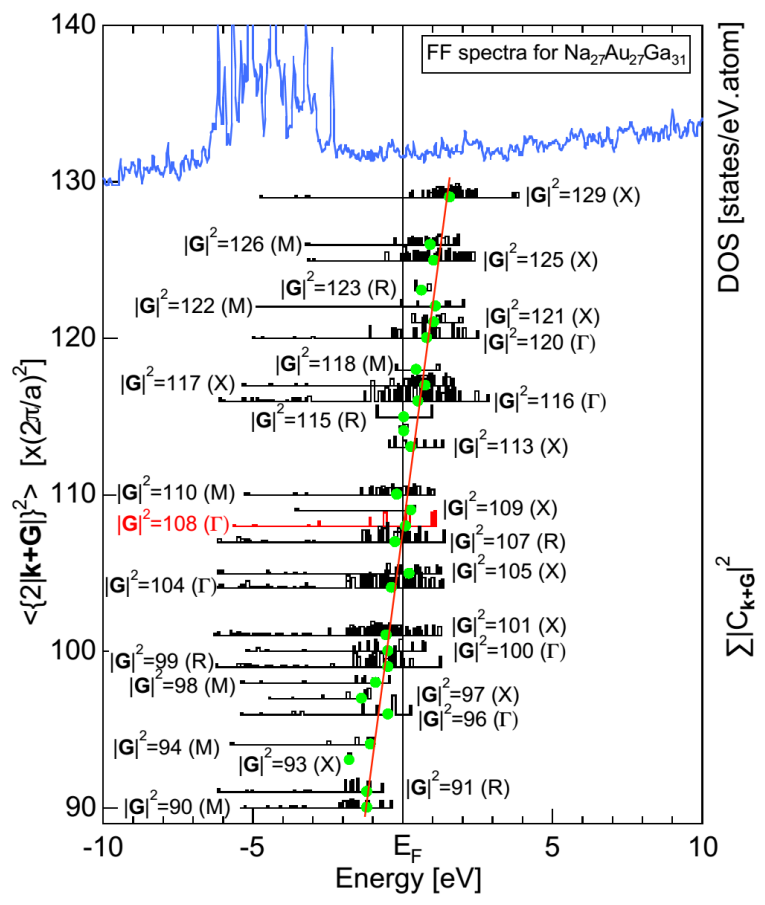

Fig. 3. FLAPW-Fourier spectrum at symmetry points $X, \quad \Gamma$, and $R$ for the 2/1-2/1-2/1 approximant $\mathrm{Na}_{27} \mathrm{Au}_{27} \mathrm{Ga}_{31}$. The total DOS is superimposed. The circle indicates the center of gravity energy in each $|\boldsymbol{G}|^{2}$ specified wave. The line connecting circles is drawn as guide to the eye.

Figure 4a shows the energy dependence of $\left\langle\left|\sum_{\boldsymbol{k}+\boldsymbol{G}}\right| \boldsymbol{C}_{\boldsymbol{k}}+\left.\boldsymbol{G}\right|_{\max } ^{2}\right\rangle_{E}$ or briefly $\left\langle|\boldsymbol{C}|_{\max }^{2}\right\rangle_{E}$, which represents the square of the maximum Fourier coefficient extracted from wave functions outside the MT spheres on a given energy surface $E(\boldsymbol{k})=E[6]$. The summation is taken over all $\boldsymbol{k}_{j}$ meshes $\left(1 \leq k_{j} \leq N_{\boldsymbol{k}}\right)$ in the Brillouin zone. Its value at the Fermi level, $\left\langle|\boldsymbol{C}|_{\max }^{2}\right\rangle_{E_{\mathrm{F}}}$ may be used as a measure to judge the itinerancy of electrons at the Fermi level. Electrons at the Fermi level are regarded as being itinerant, if $\left\langle|\boldsymbol{C}|_{\max }^{2}\right\rangle_{E_{\mathrm{F}}}>0.1[6]$. The value for $\mathrm{Na}_{27} \mathrm{Au}_{27} \mathrm{Ga}_{31}$ turns out to be 0.035 , indicating that electrons at the Fermi level are highly immobile in spite of the shallow pseudogap.

The Hume-Rothery plot and its non-dimensional standard deviation $F(E)[6]$ for $\mathrm{Na}_{27} \mathrm{Au}_{27} \mathrm{Ga}_{31}$ are shown in Fig. 4b. The data points should fall on a straight line in the free electron model. An anomaly over -6 to $-3 \mathrm{eV}$ reflects the localized $\mathrm{Au}-5 d$ band. Except for the range of the Au-5d band, the data points are found to fall on a straight line, in particular, across $E_{\mathrm{F}}$, as indicated in Fig. $4 \mathrm{~b}$. The square of the Fermi diameter $\left(2 k_{\mathrm{F}}\right)^{2}$, in units of $(2 \pi / a)^{2}$, can be read off from the intersection with $E_{\mathrm{F}}$ and deduced to be $109.2 \pm 1.0$. The application of the so called tetrahedron method, while taking the center of gravity energy in the energy distribution of the FLAPW-Fourier spectrum, has enabled us to determine the value accurately in the presence of the d-band anomaly in the Hume-Rothery plot [7]. In the present case, it is found to be $109.0 \pm 1.0$, a result that is in a

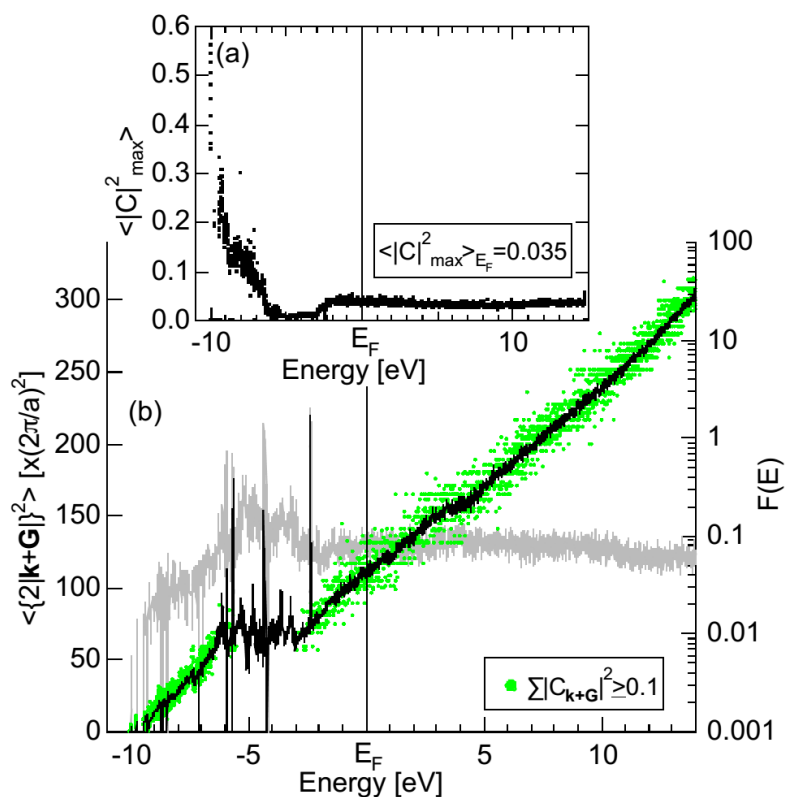

Fig. 4. (a) Energy dependence of $\left\langle|\boldsymbol{C}|_{\max }^{2}\right\rangle$ and (b) Hume-Rothery plot for the 2/1-2/1-2/1 approximant $\mathrm{Na}_{27} \mathrm{Au}_{27} \mathrm{Ga}_{31}$. Dots in (b) represent the data points satisfying the condition $\sum\left|\boldsymbol{C}_{\boldsymbol{k}}+\boldsymbol{G}\right|^{2}>0.1$ [7]. $F(E)$ in (b) represents non-dimensional standard deviation [6].

good agreement with the local reading value mentioned above.

It is clear that the interference condition $\left(2 k_{\mathrm{F}}\right)^{2}=$ $|\boldsymbol{G}|^{2}$, which plays a key role in the formation of a pseudogap at the Fermi level, is well satisfied, since $\left(2 k_{\mathrm{F}}\right)^{2}=$ 109.2 agrees well with the critical $|\boldsymbol{G}|^{2}=108$ mentioned above. We believe that the origin of the pseudogap across the Fermi level can be well explained in terms of $\mathrm{FsBz}$ interactions even in such polar intermetallics. As emphasized above, however, zones over $|\boldsymbol{G}|^{2}$ from 96 to 126 are all participating in the interference phenomenon with electrons at the Fermi level. We consider multi-zone effects to be highly intensified as a result of the polar interactions between $\mathrm{Au}$ and $\mathrm{Ga}$ atoms and to be responsible for the formation of a widely spread shallow pseudogap in the 2/1-2/1-2/1 approximant $\mathrm{Na}_{27} \mathrm{Au}_{27} \mathrm{Ga}_{31}$. It would be highly interesting to construct the FLAPW-Fourier spectrum for the isostructural but less polar $2 / 1-2 / 1-2 / 1$ approximant $\mathrm{Al}-\mathrm{Mg}-\mathrm{Zn}$ in order to study the effect of the polarity on the degree of multi-zone effects. As stated in Introduction, its atomic structure information is available in the literature [9].

The effective $e / a$ value can be immediately calculated by inserting $\left(2 k_{\mathrm{F}}\right)^{2}$ obtained above into $e / a=$ $\left\{\pi\left[\left(2 k_{\mathrm{F}}\right)^{2}\right]^{3 / 2}\right\} /(3 N)$, where $N$ is the number of atoms per unit cell and is equal to 680 . It turns out to be 1.76 in good agreement with 1.73 obtained by taking a composition average of the well-established atomic values $(e / a)_{\mathrm{Na}}=(e / a)_{\mathrm{Au}}=1.0$ and $(e / a)_{\mathrm{Ga}}=3.0[7,8]$. Hence, we can say that the strong polarity in the $2 / 1$ - 
2/1-2/1 approximant $\mathrm{Na}_{27} \mathrm{Au}_{27} \mathrm{Ga}_{31}$ does not affect the $e / a$ value at all and that the Hume-Rothery stabilization mechanism holds.

A large number of CMAs in possession of a pseudogap at $E_{\mathrm{F}}$ are found to obey the Hume-Rothery stabilization mechanism satisfying the interference condition. It is best demonstrated by plotting the number of electrons per unit cell, $e /(u c)$, being equivalent to $\left(2 k_{\mathrm{F}}\right)^{2}$ as a function of critical $|\boldsymbol{G}|^{2}$ values on a $\log -\log$ scale [8]. Figure 5 shows the $e /(u c)$ versus critical $|\boldsymbol{G}|^{2}$ diagram, into which the present data on the $2 / 1-2 / 1-2 / 1$ approximant $\mathrm{Na}_{27} \mathrm{Au}_{27} \mathrm{Ga}_{31}$ is incorporated. We see that all the data points including the present one fall on a line with a slope of $3 / 2$ in conformity with the interference condi-

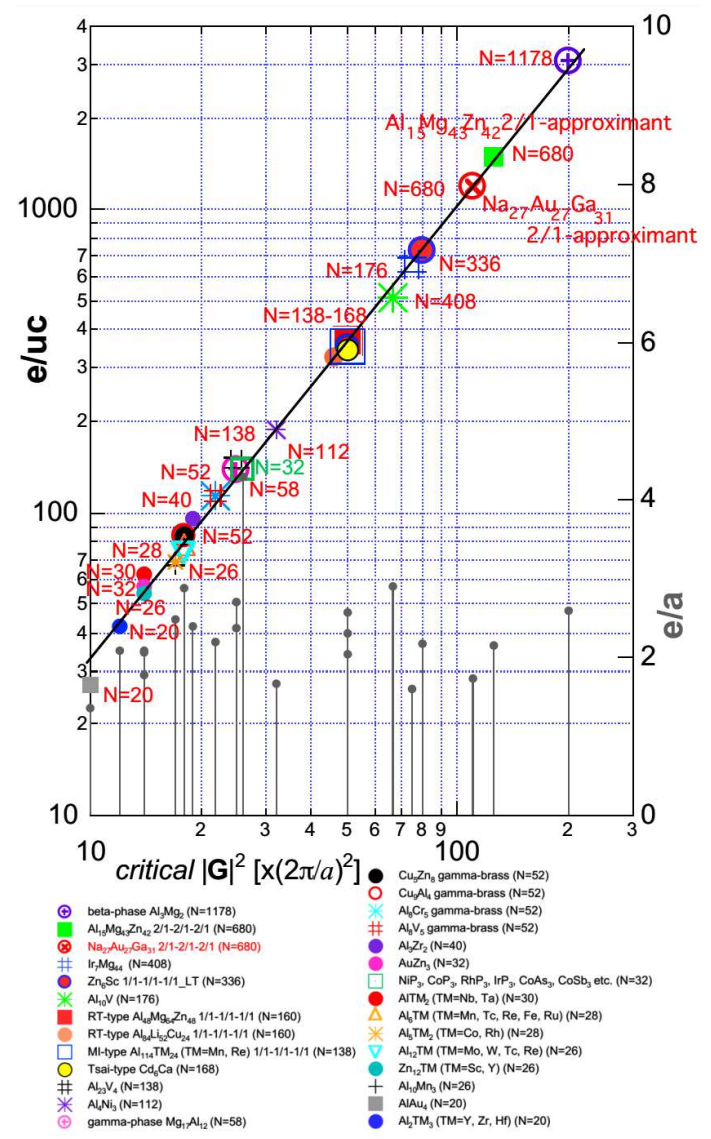

Fig. 5. $e /(u c)$ as a function of square of critical reciprocal lattice vector $|\boldsymbol{G}|_{c}^{2}$ on log-log scale for a large number of CMAs in possession of a pseudogap across the Fermi level. The $|\boldsymbol{G}|_{c}^{2}$ is expressed in units of $(2 \pi / a)^{2}$ in cubic systems. Small gray circle at the end of line represents the value of $e / a$ on the right ordinate for the respective compounds. $N$ represents the number of atoms per unit cell.

$\dagger$ The $e / a$ and critical $|\boldsymbol{G}|^{2}$ for the 2/1-2/1-2/1 approximant $\mathrm{Al}_{15} \mathrm{Mg}_{43} \mathrm{Zn}_{42}$ were estimated from a composition average of that of constituent elements, $(e / a)_{\mathrm{Al}}=3.0,(e / a)_{\mathrm{Mg}}=2.0$ and $(e / a)_{\mathrm{Zn}}=2.0$, and from X-ray diffraction spectrum, respectively, without carrying out the FLAPW band calculations $[6,8]$. tion $e /(u c)=\frac{\pi}{3}\left(|\boldsymbol{G}|^{2}\right)^{3 / 2}$. This leads us to conclude that all pseudogap-bearing CMAs, regardless of the degree of orbital hybridizations or that of the polarity, obey the Hume-Rothery stabilization mechanism.

Finally, it is worthwhile noting that the dataset for the present $\mathrm{Na}_{27} \mathrm{Au}_{27} \mathrm{Ga}_{31}$ compound is positioned lower than that of the 2/1-2/1-2/1 approximant $\mathrm{Al}_{15} \mathrm{Mg}_{43} \mathrm{Zn}_{42}$ on the $3 / 2$-power law line, though both compounds belong to the isostructural family with space group $\mathrm{Pa} \overline{3}$ and the Pearson symbol $c P 680^{\dagger}$. This is due certainly to the possession of different $e / a$ values between these two approximants with $e / a=1.76$ and 2.31 , respectively. The presence of two different $e / a$ 's is not unusual. For example, we have the $1 / 1-1 / 1-1 / 1$ approximants $\mathrm{Na}_{26} \mathrm{Au}_{24} \mathrm{Ga}_{30}(e / a=1.75)[1,2]$ and $\mathrm{Al}_{48} \mathrm{Mg}_{64} \mathrm{Zn}_{48}$ $(e / a=2.31)[3,4,6]$ with space group $\operatorname{Im} \overline{3}$ and Pearson symbol $c I 160$. The reason why more than two $e / a$ values can occur in systems with the same space group and Pearson symbol is discussed in [11]. It is of our urgent plan to determine both $\left(2 k_{\mathrm{F}}\right)^{2}$ and critical $|\boldsymbol{G}|^{2}$ by performing first-principles FLAPW band calculations for 1/1-1/1$1 / 1$ approximants $\mathrm{Na}_{26} \mathrm{Au}_{24} \mathrm{Ga}_{30}(e / a=1.75)$ and $\mathrm{Al}-$ Mg-Zn 2/1-2/1-2/1 approximant. A comparison of the FLAPW-Fourier spectrum like Fig. 3 between them will allow us to gain a deeper insight into the effect of polar interactions on the spread of $|\boldsymbol{G}|^{2}$-dependent electronic states at $E_{\mathrm{F}}$

\section{Acknowledgments}

One of the authors (U.M.) is grateful for the financial support of the Grant-in-Aid for Scientific Research (Contract No. 23560793) from the Japan Society for the Promotion of Science.

\section{References}

[1] V. Smetana, Q. Lin, D.K. Pratt, A. Kreyssig, M. Ramazanoglu, J.D. Corbett, A.I. Goldman, G.J. Miller, Angew. Chem. Int. Ed. 51, 12699 (2012).

[2] Q. Lin, V. Smetana, G.J. Miller, J.D. Corbett, Inorg. Chem. 51, 8882 (2012).

[3] U. Mizutani, Hume-Rothery Rules for Structurally Complex Alloy Phases, CRC Press, Taylor \&Francis Group, Boca Raton 2010.

[4] M. Inukai, E.S. Zijlstra, H. Sato, U. Mizutani, Philos. Mag. 91, 4247 (2011)

[5] U. Mizutani, M. Inukai, H. Sato, E.S. Zijlstra, Philos. Mag. 92, 1691 (2012).

[6] U. Mizutani, M. Inukai, H. Sato, E.S. Zijlstra, Chem. Soc. Rev. 41, 6799 (2012).

[7] H. Sato, M. Inukai, E.S. Zijlstra, U. Mizutani, Philos. Mag. 93, 3029 (2013).

[8] U. Mizutani, H. Sato, M. Inukai, E.S. Zijlstra, Philos. Mag. 93, 3353 (2013).

[9] Q. Lin, J.D. Corbett, Proc. Natl. Acad. Sci. USA 103, 13589 (2006)

[10] P. Blaha, K. Schwarz, G. Madsen, D. Kvasnicka, J. Luitz, WIEN2k, (last accessed on August 30, 2013).

[11] U. Mizutani, H. Sato, M. Inukai, E.S. Zijlstra, Acta Phys. Pol. A 126, 531 (2014). 